\title{
Changes in the frequency and in vivo vessel-forming ability of rhesus monkey circulating endothelial colony-forming cells across the lifespan (birth to aged)
}

\author{
W. Chris Shelley ${ }^{1}$, Alyssa C. Leapley ${ }^{2}$, Lan Huang ${ }^{1}$, Paul J. Critser ${ }^{1}$, Pingyu Zeng ${ }^{1}$, Daniel Prater ${ }^{2}$, David A. Ingram¹, \\ Alice F. Tarantal ${ }^{2-4}$ and Mervin C. Yoder ${ }^{1}$
}

INTRODUCTION: We have identified a novel hierarchy of human endothelial colony-forming cells (ECFCs) that are functionally defined by their proliferative and clonogenic potential and in vivo vessel-forming ability. The rhesus monkey provides an excellent model in which to examine the changes in circulating concentrations and functions of ECFCs since this nonhuman primate possesses a long lifespan and has been used extensively to model age-related processes that occur in humans.

RESULTS: Endothelial cells (ECs) derived from rhesus monkey ECFCs share a cell-surface phenotype similar to human cord blood ECFCs, rapidly form capillary-like structures in vitro, and form endothelial-lined vessels in vivo upon implantation in immunodeficient mice in an age-dependent manner. Of interest, although ECFCs from the oldest monkeys formed capillary-like structures in vitro, the cells failed to form inosculating vessels when implanted in vivo and displayed a deficiency in cytoplasmic vacuolation in vitro; a critical first step in vasculogenesis.

DISCUSSION: Utilizing previously established clonogenic assays for defining different subpopulations of human ECFCs, we have shown that a hierarchy of ECFCs, identical to human cells, can be isolated from the peripheral blood of rhesus monkeys, and that the frequency of the circulating cells varies with age. These studies establish the rhesus monkey as an important preclinical model for evaluating the role and function of circulating ECFCs in vascular homeostasis and aging.

METHODS: Peripheral blood samples were collected from 40 healthy rhesus monkeys from birth to 24 years of age for ECFC analysis including immunophenotyping, clonogenic assays, and in vivo vessel formation.

$\mathbf{T}$ he paradigms for vascular formation and repair can be generally divided into the well-recognized and extensively studied processes of vasculogenesis, angiogenesis, and arteriogenesis (1). However, postnatal vasculogenesis is a novel paradigm proposed by Asahara et al. (2) in which circulating progenitor cells of the endothelial lineage (endothelial progenitor cells, EPCs) are recruited to sites of vascular injury, ischemia, or inflammation, and these precursors participate in the regeneration, repair, or vascular remodeling process. Numerous methods have been proposed for identifying EPCs; however, no unique or specific marker has been reported for the prospective isolation of these cells from circulating blood. At present, EPCs can be isolated using flow cytometry and in vitro culture methods $(1,3,4)$, and many investigators have correlated the circulating concentration of EPCs with the presence and/or severity of cardiovascular diseases (5-7).

Using an in vitro colony-forming assay, we have identified a novel hierarchy of circulating EPCs in human umbilical cord blood and adult peripheral blood $(8,9)$. Endothelial colony-forming cells (ECFCs) that circulate in the bloodstream demonstrate robust clonal regenerative properties, display a wide variety of cell-surface molecules commonly observed in human arterial and venous endothelial cells (ECs), and demonstrate self-renewal capacity and lineage restriction and contribute only to the endothelial lineage (8-10). The ECFC progeny form in vitro capillary-like structures and spontaneously form a capillary plexus in type 1 collagen-fibronectin gels upon implantation into immunodeficient mice (10). These transplanted human capillaries inosculate with nearby endogenous murine vessels to become a part of the systemic circulation of mouse blood cells $(11,12)$. Thus, ECFCs display all the properties expected in a circulating human EPC.

The outgrowth of ECs from the peripheral blood of other mammalian species has been reported, including the rhesus monkey (13). In mice, circulating ECFCs are extremely rare and peripheral blood from more than five animals is required to ensure the growth of at least a single colony (14). Circulating ECFCs are also rare in porcine blood (1.5 colonies $/ 10 \mathrm{ml}$ ), and the number and proliferative potential have been shown to increase after an acute myocardial infarction (15). In some instances, ECFCs have been identified in cultures of ECs isolated from tissues or blood vessels (16-18). However, in most cases, the clonal proliferative potential of

'Department of Pediatrics, Herman B Wells Center for Pediatric Research, Indiana University School of Medicine, Indianapolis, Indiana; ${ }^{2}$ California National Primate Research Center, University of California-Davis, Davis, California; ${ }^{3}$ Department of Pediatrics, University of California-Davis, Davis, California; ${ }^{4}$ Department of Cell Biology and Human Anatomy, University of California-Davis, Davis, California. Correspondence: Alice F. Tarantal (aftarantal@primate.ucdavis.edu) 
ECFCs has not been rigorously tested. This is a particularly interesting point because significant differences in the proliferative potential are displayed by ECFCs from human umbilical cord and adult peripheral blood, suggesting an age-related change in the function of ECFCs (8). Studies have suggested that ECFCs from cord blood may display greater in vivo vessel-forming ability compared with cells isolated from adult peripheral blood (11).

The rhesus monkey provides an excellent model for the examination of the changes in the circulating concentration and function of ECFCs, as this nonhuman primate possesses a reasonably long lifespan ( $25 \mathrm{y}$ in captivity) and has been used extensively to model age-related processes that occur in humans. These studies suggest that the circulating concentration of ECFCs changes with age and that the proliferative potential of individual ECFC progeny and vessel-forming ability declines with age. Given the similar proliferative kinetics, circulating frequency, cell-surface phenotype, and in vivo vessel-forming ability of young monkey ECFCs to human umbilical cord blood ECFCs, these studies support that the rhesus monkey provides an invaluable model system in which to examine the role of ECFC cell therapy in treating human cardiovascular and related disease states.

\section{RESULTS}

\section{Characterization of ECFCs}

ECFC-derived EC colonies emerged from plated mononuclear cells (MNCs) at 7-20 d after the initiation of the cultures and the overall average frequency was $24 \pm 4$ colonies $/ 10^{8}$ MNCs $(N=28)$. Among all the subjects tested, cells from 12 animals in the older group $(\geq 18 \mathrm{y})$ failed to exhibit any colony growth in vitro. Differences in the total number of colonies obtained were observed in all age groups. The youngest animals (birth to $1 \mathrm{y}$ ) showed a median of 3 (range 2-25) with a frequency of $1.50 \mathrm{col}-$ onies $/ \mathrm{ml}$ of blood $(N=8)$ whereas juveniles displayed a median of 8 (range 1-31) with a frequency of 3.10 colonies $/ \mathrm{ml}$ of blood $(N=8)$. Adults indicated a median of 10 (range 2-90) with a frequency of 0.52 colonies $/ \mathrm{ml}$ of blood $(N=8)$ whereas the oldest animals ( $\geq 18 \mathrm{y})$ showed a median of 2 (range 2-4) with a frequency of 0.06 colonies $/ \mathrm{ml}$ of blood $(N=4)$. The male-tofemale ratio of 2:1 was consistent in all age groups. Thus, circulating ECFCs reached a peak in young animals and showed a significant decline in adults and aging monkeys $(P<0.05)$. All ECFC-derived EC colonies were able to expand and form a monolayer of ECs with cobblestone morphology (Figure 1a). In general, ECFCs emerged earlier (7-14 d) in the culture of blood samples from young animals $(<4$ y) compared with ECFCs (12-20 d) from the oldest animals (data not shown).

Before testing the proliferative and clonogenic potential of ECFC-derived ECs, we verified that the cell progeny from monkey peripheral blood were not contaminated with other cell types. Immunophenotyping revealed that the ECs derived from the rhesus monkey ECFCs expressed a pattern of cell-surface antigens (including CD31, CD105, CD144, CD146, and Ulex europaeus lectin binding) similar to human cells $(8,10)$ (Figure $1 b)$. The ECs also weakly expressed CD34 and VEGFR2, but failed to express the hematopoietic antigens CD14, CD45, or CD133 (Figure 1b). We have also observed that the mesenchymal cells contaminating the ECFC colonies could be readily identified by the lack of CD31, CD141, and CD144 expression; lower CD105 expression; and greater CD90 expression (data not shown). When the overall percentage of rhesus ECFC-derived ECs was analyzed for cell-surface expression in the different age groups, no significant differences from birth to adulthood were observed (data not shown).

Moreover, rhesus monkey ECFC-derived ECs uniformly ingested 488-conjugated acetylated low-density lipoprotein (Invitrogen, Carlsbad, CA) and formed capillary-like structures in Matrigel (BD Biosciences, San Jose, CA), which are characteristic of ECs (Figure 1c,d). In particular, when we quantitatively

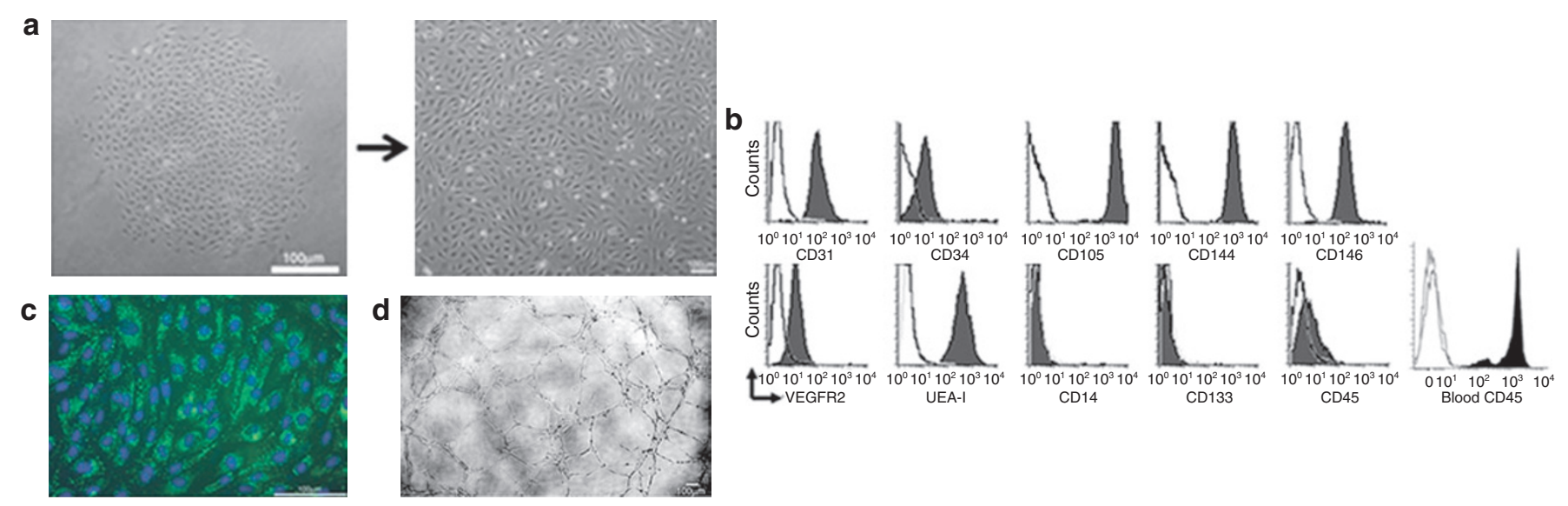

Figure 1. Phenotypic and functional analysis. (a) Representative photomicrographs of individual monkey endothelial colony-forming cell (ECFC)-derived endothelial cell (EC) colony from peripheral blood followed by EC colonies expanding to form an EC monolayer. (b) Immunophenotyping of cell monolayers from monkey peripheral blood ECFC-derived ECs by flow cytometry. ECs express CD31, CD34, CD105, CD144, CD146, VEGFR2, and Ulex europaeus agglutinin-1 (UEA-1) but not CD45, CD14, or AC133. The final panel depicts peripheral blood mononuclear cell CD45 expression as a control. (c) Monkey ECFC-derived ECs were able to ingest 488-conjugated acetylated low-density lipoprotein (green); nuclei stained with 4',6-diamidino-2-phenylindole dihydrochloride (blue). (d) Formation of capillary-like structures when monkey ECFC-derived ECs were plated on Matrigel. Bar $=100 \mu \mathrm{m}$. Representative data from 28 independent experiments utilizing different animals with similar results (at least one analysis of each of 28 animals) are shown. 
analyzed the ability of ex vivo tube formation we found that there was no statistical difference between the age groups (data not shown). Thus, these analyses confirmed that the cell progeny from monkey circulating ECFCs were endothelial in origin and not contaminated with hematopoietic or mesenchymal cells.

\section{Clonogenic and Proliferative Potential}

A single-cell colony-forming assay has been used to quantitate the clonogenic potential of individual ECs derived from the human circulating ECFCs (8), and a complete hierarchy of human peripheral blood, cord blood, or vessel wall-derived ECFCs has been identified that is composed of high-proliferative-potential ECFCs (HPP-ECFCs), low-proliferative-potential ECFCs, endothelial cluster cells, and nondividing mature ECs $(8,9)$. ECs derived from human umbilical cord ECFCs display greater proliferative potential than those from adult peripheral blood, suggesting an age-related change in the function of ECFCs. We used this method to investigate whether there was a hierarchy of proliferative potential in ECFCs present in rhesus monkey peripheral blood and whether the proliferative potential may be altered with age.

Plating single circulating ECFC-derived ECs resulted in a diverse display of differences in proliferative ability. Significantly more single cells divided in the youngest animals (birth to $<4$ y) when compared with the older animals ( 4 to $<18$ y; Figure 2 ). Of the single cells that divided, heterogeneity in the proliferative potential was observed that varied with age (Figure 2). The progeny of some single-plated ECs were able to give rise to $>2,000$ progeny and were capable of being replated to form secondary and tertiary colonies of the same size, thus demonstrating the properties of derivation from an HPP-ECFC (data not shown) as previously described (8). Therefore, a complete hierarchy of ECFCs is present in the peripheral blood in rhesus monkeys as in human subjects. Notably, when the youngest animals were compared with the adults, there were significantly more plated single cells in 4 - to $<18$-y-old adult animals that divided but gave rise to small colonies containing $<50$ progeny (infants $42 \pm 7 \%$ vs. adults $84 \pm 13 \%)$. By contrast, the percentage of single cells giving rise to colonies of $>10,000$ cells (HPP-ECFC) dramatically decreased in the adult and aging animals (Figure 2). Thus, the distribution of ECFCs was skewed toward HPP-ECFC in the young animals but weighted toward minimally proliferative endothelial cluster-forming cells or low-proliferative-potential ECFCs in the adult and aging animals. Simple linear regression analysis was conducted to determine whether there was a relationship with age; the results demonstrated that the percentage of dividing cells and colonies containing $>10,000$ cells decreased significantly with increasing age $(P<0.05)$ and the percentage of colonies containing both $2-50$ and $51-200$ cells $(P<0.05)$ increased with advancing age. Regression analysis suggested that the proliferative potential of ECFCs is exhausted as the animal progresses through its lifespan.

\section{Chimeric Blood Vessel Formation}

We $(10)$ and others $(11,12,19)$ have reported that human ECFCs possess the potential to form de novo blood vessels when transplanted subcutaneously in a collagen-fibronectin matrix of immunodeficient mice. Further, cord blood-derived ECFCs have been shown to have a greater potential to form de novo blood vessels in vivo than ECFCs derived from adult peripheral blood $(11,12)$. Using the same methods, we investigated whether there was an age-dependent difference in the vessel-forming potential of monkey ECFCs over the lifespan. Although coimplantation of mesenchymal stromal cells or adipose stromal cells is known to enhance the in vivo vessel-forming ability of the human cord blood and adult peripheral blood ECFCs $(11,20)$, we chose not to include these supportive cells in the scaffolds in the present studies to more directly and rigorously assess the vasculogenic potential of the rhesus ECFCs in vivo.

ECFCs from monkeys of varying ages were suspended in a collagen-fibronectin matrix and transplanted into immunodeficient mice. At $14 \mathrm{~d}$, mice were euthanized, and the grafts were
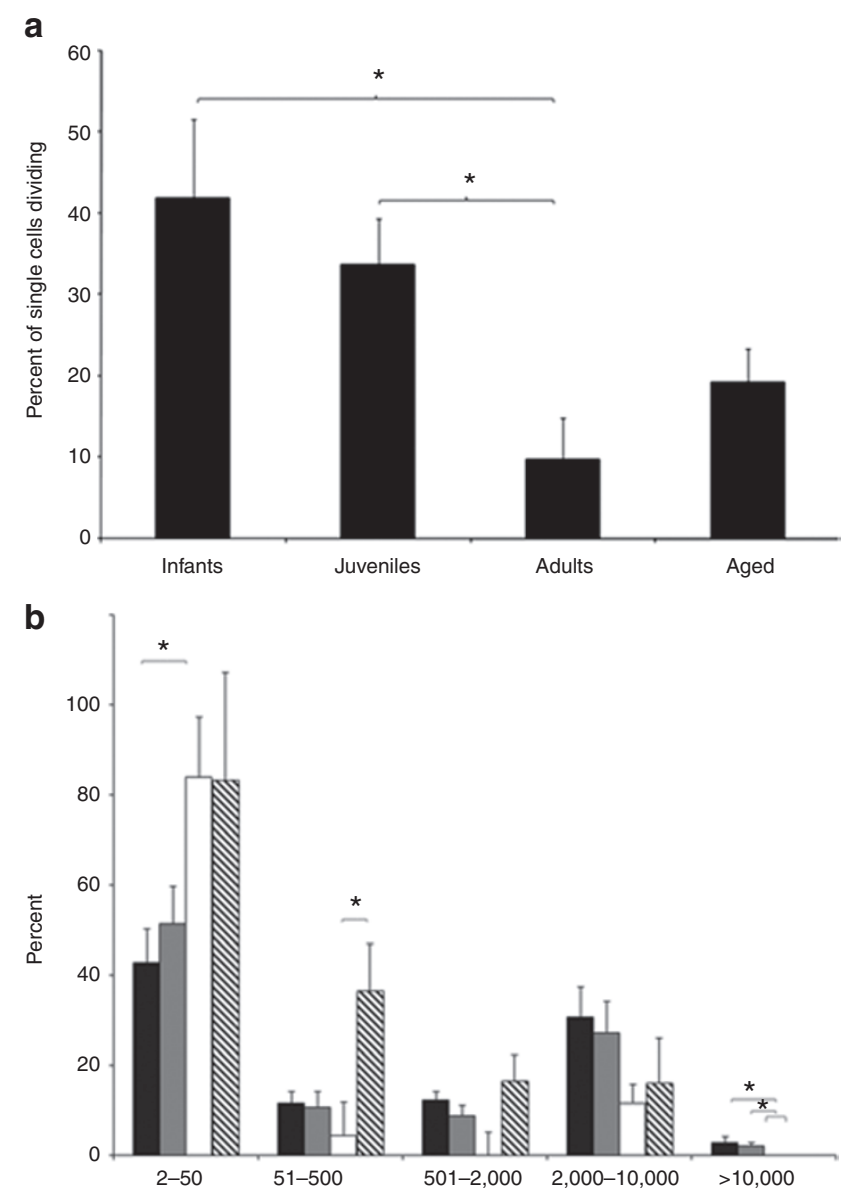

Figure 2. Quantitation of clonogenic and proliferative potential of endothelial cells (ECs) derived from rhesus monkey peripheral blood. (a) The percentage of single endothelial colony-forming cell (ECFC)-derived ECs dividing at least once after $14 \mathrm{~d}$ in culture is shown. Fewer cells divided in the adult age group ( 4 to $<18 \mathrm{y}$ ) compared with the younger animals. (b) The distribution of colonies of different size derived from a single EC in an individual well after $14 \mathrm{~d}$ of culture is represented. There was a dramatically higher percentage of cells that formed high-proliferative-potential ECFC colonies in the animals $<4$ y of age compared with adults. ${ }^{*} P<0.05$, by parametric ANOVA ( $N=8 /$ group). Bars represent the age of the animals:

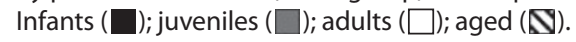




\section{Shelley et al. Articles}
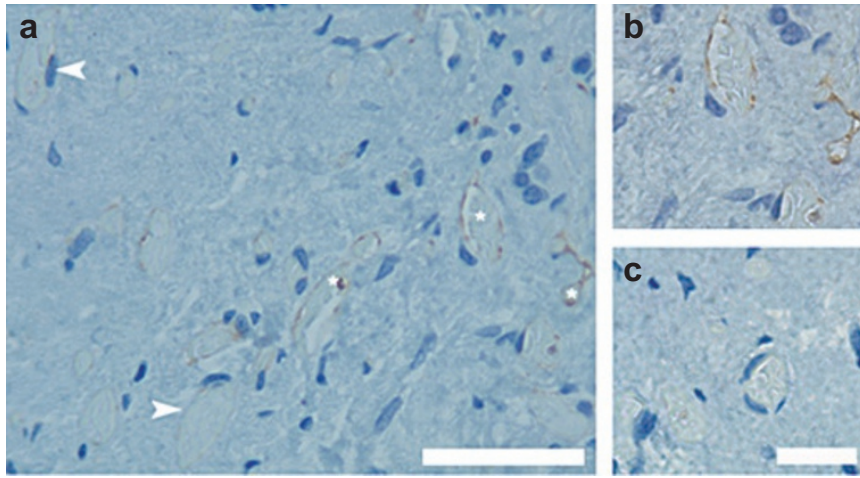

Figure 3. Rhesus monkey endothelial colony-forming cells demonstrate the potential to form functional capillaries in vivo. (a) Anti-CD31-labeled monkey vessels $\left(^{*}\right)$ containing mouse red blood cells (RBCs) and mouse vessels (arrowhead) are shown. (b) Higher magnification of anti-CD31labeled monkey capillary containing mouse RBCs. (c) Higher magnification of mouse capillary that did not stain with the anti-CD31 antibody. Bar = $100 \mu \mathrm{m},(\mathbf{a}) ; 50 \mu \mathrm{m},(\mathbf{b}, \mathbf{c})$ (representative data from 4 subjects/group implanted into a total of 16 recipient mice).

harvested and analyzed for chimeric blood vessel formation. The grafts were stained for antihuman CD31 antibodieswhich cross-react with monkey but not mouse-to differentiate between host and donor vasculature. The grafts were then examined for functional monkey-derived capillaries determined by the expression of CD31 and the presence of mouse red blood cells within the vessel. In all the experiments, ECFCs from the younger animals (birth to $<4 \mathrm{y}$ ) and adults $(4$ to $<18$ y) were able to form functional capillaries (Figure 3). However, ECFCs from the oldest animals failed to form functional chimeric vessels, suggesting a loss of ECFCs' vessel-formation ability with aging (data not shown).

\section{Cytoplasmic Vacuolation In vitro}

We have assessed in vivo vasculogenesis by placing rhesus monkey ECFCs in collagen scaffolds and implanting the gels into host immunodeficient mice in the absence of any coimplanted stromal supportive cells. To begin to address the mechanism(s) that led to the failure of the ECFCs from aged monkeys to form vessels in vivo, we tested the ability of young and old rhesus monkey ECFCs to undergo cytoplasmic vacuolation in vitro, given that this process is known to represent the fundamental step in EC formation of lumenized structures in a three-dimensional gel in vitro and in vivo $(21,22)$. Vacuole density and total vascular area were significantly increased in matrices seeded with rhesus ECFCs from juvenile monkeys compared with aged monkeys (>18 y) (Figure 4). There was also a trend toward an increase in average vacuole area in matrices seeded with ECFCs from juvenile monkeys compared with aged animals. No differences in the level of apoptosis of the implanted ECFCs within the gels among the study groups were noted (data not shown). Although this in vitro assay examines only the first critical step in vasculogenesis, it has been determined that blocking this step is sufficient to prevent in vivo vessel formation in several vertebrate model systems $(21,22)$.
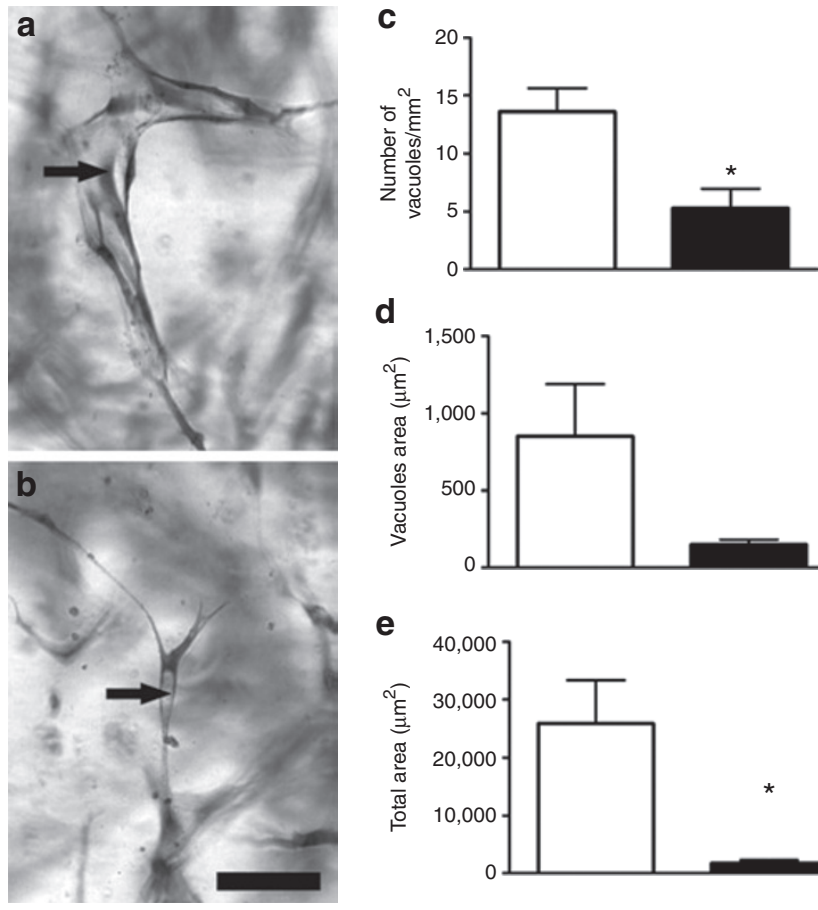

d
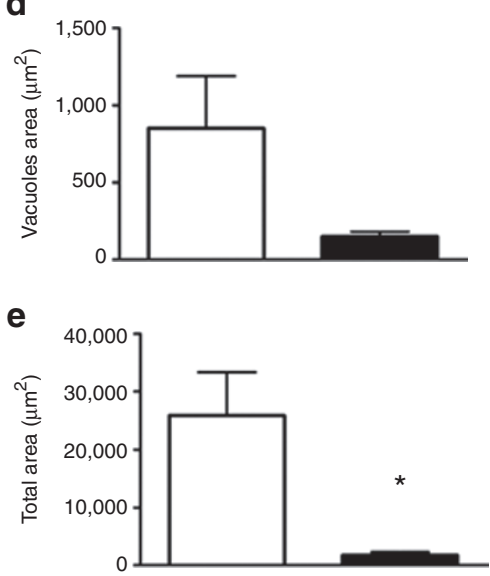

Figure 4. Rhesus endothelial colony-forming cells (ECFCs) display varying ability to form vacuoles in vitro based on age. (a) Juvenile and (b) aged monkey ECFCs were able to form vacuoles in vitro (arrows indicate vacuoles). Bar $=100 \mu \mathrm{m}$. Younger ECFCs (white bars) demonstrated an increased (c) vacuole density, (d) vacuole area, and (e) total vacuole area compared with $>18$-y-old monkeys (black bars) that formed vacuoles $\left({ }^{*} P<0.05 ;\right.$ three individuals from each group were analyzed).

\section{DISCUSSION}

Although EPCs have previously been isolated from rhesus monkey peripheral blood and vessels (13), their phenotype and function have not been completely identified. In the present study, we demonstrated that a complete hierarchy of ECFCs exists in the peripheral blood of the rhesus monkey as previously shown in humans $(8,9)$. Further, monkey ECFCs display the potential to form functional vessels upon in vivo implantation in nonobese diabetic/severe combined immunodeficient mice.

The frequency and blood vessel-forming potential of human ECFCs have been shown to decrease in adult peripheral blood when compared with umbilical cord blood ECFCs $(11,19)$, suggesting an effect of aging. To determine whether a similar effect is seen in the rhesus monkey, we evaluated the frequency, proliferative potential, and vessel-forming capabilities in circulating ECFCs over the monkey lifespan. This study demonstrated that the circulating concentration of ECFCs changes with age, and that the proliferative potential and the vessel-forming ability of ECFC progeny declines with age. This is the first study to report such changes over the lifespan of an organism.

Rhesus ECFCs display an EPC phenotype that is stable across all age groups. Similar to human ECFCs, monkey ECFCs express EC-specific markers CD31, CD105, CD144, CD146, and VEGFR2 and can take up the plant lectin Ulex europaeus agglutinin-1. Importantly, monkey ECFCs do not express hematopoietic cell markers such as CD45 and CD14. Further, 
monkey ECFCs displayed the ability to ingest acetylated lowdensity lipoprotein in culture and form capillary structures $e x$ vivo on Matrigel. This is consistent with the previous reports characterizing rhesus monkey EPCs (13) but extends the prior observations to both younger and older animals.

Although the ECFC phenotype was consistent across the monkey lifespan, the frequency of circulating ECFCs changed with age. The average frequency of all samples tested was $24 \pm 4$ colonies/100 million MNCs, which is consistent with prior published data (13). However, the frequency of ECFC colonies was greatest in young animals, remained somewhat stable in adults, and dramatically declined with aging.

Human ECFCs from umbilical cord blood demonstrate a higher proliferative potential compared with adult peripheral blood-derived ECFCs. Further, a single-cell clonogenic assay demonstrated that the distribution of ECFCs in cord blood is skewed toward HPP-ECFCs, and this was shown to be accompanied by an increase in telomerase activity in cord bloodderived ECFCs (8). The lack of adequate ECFC proliferation in colonies from the aged monkeys prohibited determination of telomerase activity in this study; however, identification of a significant difference in the ability of the ECFCs from the aged monkeys to undergo cytoplasmic vacuolation highlights a potential functional target for more investigation to understand this age-related finding. Although ECFCs from juvenile $(1-4 \mathrm{y})$ and a subset of aging monkeys (>18 y) displayed the ability to form vacuoles in vitro, the ECFCs from juveniles were able to form vacuoles to a significantly greater degree. The increase in both vacuole density and total vacuole area suggests that juvenile monkey ECFCs are more readily able to respond to the extracellular matrix cues necessary for ECs to undergo vasculogenesis than are ECFCs from older animals. Given that vacuole formation is proposed to be an initiating step in vessel formation $(21,22)$, this reduced ability to undergo vacuolization in vitro is a potential mechanism for the inability of ECFCs from older animals to form functional vessels in vivo.

The current study demonstrates that the change in proliferative capacity of rhesus monkey ECFCs across the lifespan is consistent with results previously demonstrated in humans and that the aged monkeys display differences in the initial steps of vasculogenesis and are unable to form vessels in vivo. These findings suggest that the rhesus monkey is an important model system with which to further study the effects of aging and cardiovascular disease on the biology of human circulating cells that are fated to assist in vessel repair and regeneration.

\section{METHODS}

\section{Animals}

Peripheral blood samples $(5-40 \mathrm{ml})$ were collected from 40 healthy rhesus monkeys (Macaca mulatta) from birth to $24 \mathrm{y}$ of age. Groups included infant (newborns to $1 \mathrm{y}$ ), juvenile ( 1 to $<4$ y; puberty $~ 3-4 \mathrm{y}$ ), adult (4-18 y), and aged monkeys ( $\geq 18-24 \mathrm{y})$. All procedures conformed to the requirements of the Animal Welfare Act, and protocols were approved before implementation by the Institutional Animal Care and Use Committee at the University of California-Davis (monkey) and at Indiana University (mice; $N=6$ ).

\section{MNC Isolation}

Monkey MNCs were obtained as previously described with minor modifications (23). Blood was diluted 1:1 with Hank's Balanced Salt Solution (Invitrogen) and overlaid onto an equivalent volume of Histopaque 1077 (Sigma, St Louis, MO). Cells were centrifuged for $30 \mathrm{~min}$ at room temperature at $740 \mathrm{~g}$. MNCs were isolated and washed three times with EC growth medium-2 (EGM-2; Lonza, Walkersville, MD) supplemented with $10 \%$ fetal bovine serum (Hyclone, Logan, $\mathrm{UT}$ ), $2 \%$ penicillin/streptomycin (Invitrogen), and $0.25 \mu \mathrm{g} / \mathrm{ml}$ of amphotericin B (Invitrogen; complete EGM-2).

\section{EC Culture}

MNCs were seeded at a density of 3-5 $\times 10^{7}$ cells/well in $4 \mathrm{ml}$ of complete EGM-2 in six-well tissue culture plates precoated with type I rat tail collagen (BD Biosciences) at $37^{\circ} \mathrm{C}, 5 \% \mathrm{CO}_{2}$ in a humidified incubator. After $24 \mathrm{~h}$, nonadherent cells and debris were aspirated, adherent cells washed with complete EGM-2, and complete EGM-2 added to each well. Medium was changed daily for $7 \mathrm{~d}$ and then every other day until the first passage.

EC colonies appeared between 7 and $20 \mathrm{~d}$ of culture and were identified as well-circumscribed, adherent colonies of cobblestone-appearing cells. The EC colonies from ECFCs were enumerated by visual inspection with an inverted microscope (Olympus, Lake Success, NY). The ECs derived from the colonies were released from the original tissue culture plates utilizing trypsin-EDTA (Invitrogen), resuspended in complete EGM-2, and plated onto $75-\mathrm{cm}^{2}$ tissue culture flasks coated with type I rat tail collagen for further passage.

\section{Matrigel Assays}

To assess the ability of the cells to incorporate acetylated low-density lipoprotein, the attached cells were incubated with $10 \mu \mathrm{g} / \mathrm{ml}$ of 488-conjugated acetylated low-density lipoprotein (Invitrogen) in the medium for $4 \mathrm{~h}$ at $37^{\circ} \mathrm{C}$. Cells were washed three times, stained with $1.5 \mu \mathrm{g} / \mathrm{ml}$ of $4^{\prime}, 6$-diamidino-2-phenylindole dihydrochloride (Sigma), and examined through an inverted fluorescence microscope (Zeiss, Thornwood, NY).

Matrigel assays were performed as previously described (8). Early-passage (2-3) ECFC-derived ECs were seeded onto 96-well tissue culture plates coated with $30 \mu \mathrm{l}$ of Matrigel (BD Biosciences) at a cell density of 5-20 $\times 10^{3}$ cells/well. Cells were observed every $2 \mathrm{~h}$ with an inverted microscope for the formation of capillary-like structures. The total length of capillary-like structures was quantitated using Image J software (NIH, Bethesda, MD) as previously described (8).

\section{Immunophenotyping}

Early-passage $(1-2)$ ECFC-derived ECs $\left(5 \times 10^{5}\right)$ were incubated at $4^{\circ} \mathrm{C}$ for $30 \mathrm{~min}$ with varying concentrations of the primary or isotype control antibody in $100 \mu$ l of phosphate-buffered saline and $2 \%$ fetal bovine serum, washed three times, and analyzed by fluorescence-activated cell sorting (BD Biosciences). Primary murine monoclonal antibodies (BD Biosciences) were used against human CD31 conjugated to fluorescein isothiocyanate (clone WM59), antihuman CD34 conjugated to phycoerythrin (PE; clone 563), antihuman VEGFR2 conjugated to fluorescein isothiocyanate (clone 89106), antihuman CD14 conjugated to PE (clone MrE2), antihuman CD45 conjugated to fluorescein isothiocyanate (clone HI30), antihuman CD146 conjugated to PE (clone P1H12), antihuman AC133 conjugated to PE (clone JM3), antihuman CD105 conjugated to PE (clone 266), and antihuman CD144 (clone 55-7H1) conjugated to Alexa Fluor 647 (Molecular Probes, Eugene, OR). Isotype control antibodies of the IgG1 and IgG2A subclass were used. All antibodies have been previously validated to cross-react with appropriate cell subsets in the rhesus monkey. For detection of the ability to bind to Ulex europaeus agglutinin-1, ECs were stained with fluorescein isothiocyanate-conjugated Ulex europaeus agglutinin-1 (Vector Laboratories, Burlingame, CA).

\section{Single-Cell Clonogenic Assays}

For early-passage (2-4) ECFC-derived ECs, a fluorescence-activated cell sorting Vantage Sorter (BD Biosciences) was used to place one cell/well into 96-well plates precoated with type I rat tail collagen in 
$200 \mu$ l of complete EGM-2. Individual wells were examined under a fluorescence microscope to ensure that only one cell was placed into each well. Cells were cultured at $37^{\circ} \mathrm{C}, 5 \% \mathrm{CO}$ in a humidified incubator. Media were changed every $5 \mathrm{~d}$. After $14 \mathrm{~d}$ of culture, the cells were fixed with $4 \%$ paraformaldehyde (Sigma) in phosphate-buffered saline for $30 \mathrm{~min}$ at room temperature, then washed twice, stained with $1.5 \mu \mathrm{g} / \mathrm{ml} \mathrm{4}$ ',6-diamidino-2-phenylindole dihydrochloride, and examined for EC growth. Wells containing $\geq 2$ cells were identified as positive for proliferation and wells containing $<50$ cells were counted. For wells with $>50$ cells, colonies were imaged and cell number quantified using version 1.36 of the Image J program (developed by Wayne Rasband, NIH).

\section{In vivo Assays}

Early-passage (3-5) ECFC-derived ECs $\left(2 \times 10^{6}\right.$ cells $\left./ \mathrm{ml}\right)$ were suspended in a $1.5 \mathrm{mg} / \mathrm{ml}$ collagen-fibronectin matrix as previously described (10). Volumes $(1 \mathrm{ml})$ were pipetted into wells of 12 -well plates, allowed to polymerize at $37^{\circ} \mathrm{C}$ for $30 \mathrm{~min}$, and covered with complete EGM-2 for overnight incubation at $37^{\circ} \mathrm{C}, 5 \% \mathrm{CO}$. After $18 \mathrm{~h}$ of culture, cellularized matrices were bisected and implanted into the flanks of 6- to 8-wk-old nonobese diabetic/severe combined immunodeficient mice as previously described (10). After $14 \mathrm{~d}$, the grafts were harvested, fixed in formalin-free zinc fixative (BD Biosciences), paraffin embedded, bisected, and sectioned $(6 \mu \mathrm{m})$ for analysis by immunohistochemistry $(N=6)$.

\section{Immunohistochemistry}

Sections were stained as previously described (11). Briefly, paraffinembedded tissue sections were deparaffinized and immersed in retrieval solution (Dako, Carpenteria, CA) for $20 \mathrm{~min}$ at $90-99^{\circ} \mathrm{C}$. Slides were incubated at room temperature for $30 \mathrm{~min}$ with antihuman CD31 (clone JC70/A; Abcam, Cambridge, UK), followed by 10-min incubation with LASB2 link-biotin and streptavidin-horseradish peroxidase (Dako), then developed with 3,3'-diaminobenzidine solution (Vector Laboratories, Burlingame, CA) for $5 \mathrm{~min}$.

\section{Cytoplasmic Vacuolation Analysis}

ECFCs were suspended in collagen solutions at a cell density of $2 \times 10^{6}$ cells/ml before polymerization to ensure uniform distribution throughout the type I collagen matrix. The matrix was polymerized for $30 \mathrm{~min}$ at $37^{\circ} \mathrm{C}$ and then $120 \mu \mathrm{l}$ of warm EGM-2 (Lonza, Basel, Switzerland)

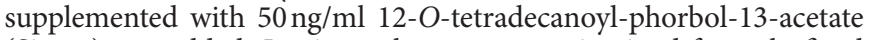
(Sigma) was added. In vitro cultures were maintained for $48 \mathrm{~h}$, fixed with $4 \%$ paraformaldehyde, and then stained with $0.1 \%$ toluidine blue $\mathrm{O}$ in $30 \%$ methanol. Bright-field microscopy was used to visualize ECFC-derived vascular structures. Nine fields of view were analyzed using a standard image-analysis system (Metamorph; Molecular Devices, Sunnyvale, CA). Total vacuole area was determined via summation of all vacuole areas scored.

\section{Statistical Analysis}

Results are shown as the mean \pm SEM. Data were analyzed with ANOVA, and significant differences were identified $(P<0.05)$ using GraphPad InStat software (La Jolla, CA). To test whether there was a relationship between age and proliferative potential, simple linear regression was conducted using SAS statistical software (SAS Institute, Cary, NC; $P<0.05)$.

\section{STATEMENT OF FINANCIAL SUPPORT}

These studies were supported by the NIH Center of Excellence in Translational Human Stem Cell Research (P50 HL085036), the Center for Fetal Monkey Gene Transfer for Heart, Lung, and Blood Diseases (R24 HL085794), the California National Primate Research Center base operating grant (P51 RR00169), and the Riley Children's Foundation.

\section{REFERENCES}

1. Hirschi KK, Ingram DA, Yoder MC. Assessing identity, phenotype, and fate of endothelial progenitor cells. Arterioscler Thromb Vasc Biol 2008; 28:1584-95.
2. Asahara T, Murohara T, Sullivan A, et al. Isolation of putative progenitor endothelial cells for angiogenesis. Science 1997;275:964-7.

3. Povsic TJ, Zavodni KL, Vainorius E, Kherani JF, Goldschmidt-Clermont PJ, Peterson ED. Common endothelial progenitor cell assays identify discrete endothelial progenitor cell populations. Am Heart J 2009;157:335-44.

4. Timmermans F, Plum J, Yoder MC, Ingram DA, Vandekerckhove B, Case J. Endothelial progenitor cells: identity defined? J Cell Mol Med 2009; 13:87-102.

5. Kawamoto A, Losordo DW. Endothelial progenitor cells for cardiovascular regeneration. Trends Cardiovasc Med 2008;18:33-7.

6. Michowitz Y, Goldstein E, Wexler D, Sheps D, Keren G, George J. Circulating endothelial progenitor cells and clinical outcome in patients with congestive heart failure. Heart 2007;93:1046-50.

7. Fadini GP, Sartore S, Albiero M, et al. Number and function of endothelial progenitor cells as a marker of severity for diabetic vasculopathy. Arterioscler Thromb Vasc Biol 2006;26:2140-6.

8. Ingram DA, Mead LE, Tanaka $\mathrm{H}$, et al. Identification of a novel hierarchy of endothelial progenitor cells using human peripheral and umbilical cord blood. Blood 2004;104:2752-60.

9. Ingram DA, Caplice NM, Yoder MC. Unresolved questions, changing definitions, and novel paradigms for defining endothelial progenitor cells. Blood 2005;106:1525-31.

10. Yoder MC, Mead LE, Prater D, et al. Redefining endothelial progenitor cells via clonal analysis and hematopoietic stem/progenitor cell principals. Blood 2007;109:1801-9.

11. Au P, Daheron LM, Duda DG, et al. Differential in vivo potential of endothelial progenitor cells from human umbilical cord blood and adult peripheral blood to form functional long-lasting vessels. Blood 2008;111:1302-5.

12. Melero-Martin JM, De Obaldia ME, Kang SY, et al. Engineering robust and functional vascular networks in vivo with human adult and cord bloodderived progenitor cells. Circ Res 2008;103:194-202.

13. Hu J, Takatoku M, Sellers SE, et al. Analysis of origin and optimization of expansion and transduction of circulating peripheral blood endothelial progenitor cells in the rhesus macaque model. Hum Gene Ther 2002; 13:2041-50.

14. Somani A, Nguyen J, Milbauer LC, Solovey A, Sajja S, Hebbel RP. The establishment of murine blood outgrowth endothelial cells and observations relevant to gene therapy. Transl Res 2007;150:30-9.

15. Huang L, Hou D, Thompson MA, et al. Acute myocardial infarction in swine rapidly and selectively releases highly proliferative endothelial colony forming cells (ECFCs) into circulation. Cell Transplant 2007;16:887-97.

16. Alvarez DF, Huang L, King JA, ElZarrad MK, Yoder MC, Stevens T. Lung microvascular endothelium is enriched with progenitor cells that exhibit vasculogenic capacity. Am J Physiol Lung Cell Mol Physiol 2008;294:L419-30.

17. Huang L, Harkenrider M, Thompson M, et al. A hierarchy of endothelial colony-forming cell activity displayed by bovine corneal endothelial cells. Invest Ophthalmol Vis Sci 2010;51:3943-9.

18. Schniedermann J, Rennecke M, Buttler K, et al. Mouse lung contains endothelial progenitors with high capacity to form blood and lymphatic vessels. BMC Cell Biol 2010;11:50.

19. Melero-Martin JM, Khan ZA, Picard A, Wu X, Paruchuri S, Bischoff J. In vivo vasculogenic potential of human blood-derived endothelial progenitor cells. Blood 2007;109:4761-8.

20. Traktuev DO, Prater DN, Merfeld-Clauss S, et al. Robust functional vascular network formation in vivo by cooperation of adipose progenitor and endothelial cells. Circ Res 2009;104:1410-20.

21. Kamei M, Saunders WB, Bayless KJ, Dye L, Davis GE, Weinstein BM. Endothelial tubes assemble from intracellular vacuoles in vivo. Nature 2006;442:453-6.

22. Sacharidou A, Koh W, Stratman AN, Mayo AM, Fisher KE, Davis GE. Endothelial lumen signaling complexes control 3D matrix-specific tubulogenesis through interdependent Cdc42- and MT1-MMP-mediated events. Blood 2010;115:5259-69.

23. Lee CC, Fletcher MD, Tarantal AF. Effect of age on the frequency, cell cycle, and lineage maturation of rhesus monkey (Macaca mulatta) CD34+ and hematopoietic progenitor cells. Pediatr Res 2005;58:315-22. 